\title{
Stability Analysis of Pneumatic Cabin Pressure Regulating System with Complex Nonlinear Characteristics
}

\author{
Xinhua Zheng, Lili Xie, and Lizhuo Liu \\ School of Automation, Northwestern Polytechnical University, Xi'an 710129, China \\ Correspondence should be addressed to Xinhua Zheng; parisz@163.com
}

Received 26 October 2014; Accepted 11 February 2015

Academic Editor: Petko Petkov

Copyright ( 2015 Xinhua Zheng et al. This is an open access article distributed under the Creative Commons Attribution License, which permits unrestricted use, distribution, and reproduction in any medium, provided the original work is properly cited.

\begin{abstract}
Stability of pneumatic cabin pressure regulating system with complex nonlinear characteristics is considered. The mathematical model of each component is obtained and given in detail. The governing equations of the considered system consist of 8 differential equations. In the circumstance, commonly used methods of nonlinear system analysis are not applicable. Therefore a new method is proposed to construct phase plane trajectories numerically. The calculation steps are given in detail. And convergence region of numerical calculation and limits on step size is defined. The method is applied constructing phase plane trajectories for considered cabin pressure regulating system. Phase plane analysis shows that there exists a limit cycle, which is responsible for pressure pulsating in aircraft cabin. After parameters adjustment, excellent stability characteristics are acquired. And the validity of this method is confirmed by the simulation.
\end{abstract}

\section{Introduction}

It is known that cabin pressure regulating system is used to provide a safe and comfortable surrounding for crew and passengers at high altitude [1]. Now cabin pressure regulating system is necessary for commercial airplanes and military aircrafts cruising at high altitude. For commercial airplanes, digital pressurization systems had become a new developing trend. And in some newly designed airplanes, it is integrated with other systems [2]. The implement of this new technology advantages improvement in intellectualization and automation of control. At the same time, it improves comfort of the passenger and reduces labor cost in maintenance work. But these numerical systems are electrical energy dependent and sensitive to electromagnetic interference. Therefore in military aircrafts, pneumatic cabin pressure regulating systems are still preferable.

The cabin pressure regulating system considered in present paper is an all pneumatic system. It regulates cabin pressure automatically and no human interference is needed. It works pneumatically and needs no electrical energy. This makes it a system of high reliability, even if electromagnetic interference of high intensive cannot have any influence on its performance. And it adapts automatically for use on high altitude airports. It also has additional desirable characteristics of light weight, small volume, and low maintenance. These characteristics satisfy exactly the needs of advanced military airplanes. Now it is used widely in newly developed military airplanes in China.

But at certain flying conditions, pulsating of cabin pressure arises. The pulsating of cabin pressure is undesirable for it may lead to common symptoms of airplane ear including hearing loss, discomfort in the ear, or mild to moderate pain. Continuous existence of such symptoms distracts attention of pilots and reduces their work efficiency or even leads to permanent hearing impairment [3]. Therefore the stability of cabin pressure must be ensured. The main object of the present paper is to define the cause of the cabin pressure pulsating and eliminate it.

\section{Cabin Pressure Regulating System and Its Dynamic Equation}

2.1. Diagram of Cabin Pressure Regulating System. As Figure 1 displays, the considered cabin pressure regulating system 


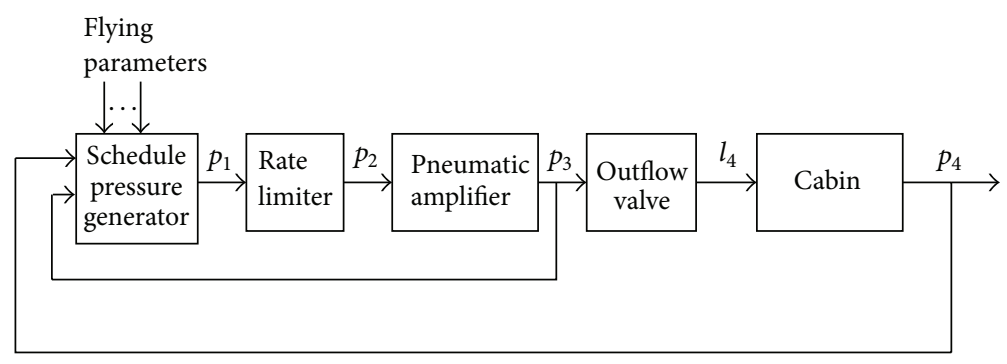

FIGURE 1: The diagram of cabin pressure regulating system.

comprises five main parts, schedule pressure generator, rate limiter, pneumatic amplifier, outflow valve, and cabin. Comprised of two independent components, schedule pressure generator is the core of the system. Its main function is to produce cabin pressure schedule signal to control outflow valve. The rate limiter limits maximum rising rate and maximum dropping rate of scheduled pressure. The output signal of rate limiter is too weak to drive pneumatic outflow valve; therefore pneumatic amplifier is used to amplify its driven capability. And as a pneumatically sourced element, it is capable of driving outflow valve directly. Outflow valve is the actuator of the system. Its function is to open valve in accordance with pressure in its control chamber, which is a pneumatic load of amplifier. At certain altitude, the flow rate of cabin air to ambient circumstance is directly proportional to the opening of outflow valve. And by this means cabin pressure is regulated.

2.2. Mathematical Model of Components. The differential equations of the cabin pressure regulating system are built on basis of the following assumptions [4].

(1) The cabin temperature $T_{p}$ keeps invariant, and $T_{p}=$ $20^{\circ} \mathrm{C}$.

(2) The cabin volume keeps constant.

(3) The air filling the cabin is ideal gas.

(4) The leakage area of the cabin is constant.

Because the cabin temperature keeps constant, the flow quantity can be measured in volume in the mathematical model built for each component. This greatly simplifies computation.

In the following mathematical models, $p_{1}, p_{2}, p_{3}$, and $p_{4}$ are the output pressures of schedule pressure generator, rate limiter, pneumatic amplifier, and cabin, $l_{1}$ is the opening of valve 1 in the active component of schedule pressure generator, $l_{31}, l_{32}$ are the opening of valve 31 and valve 32, which connect pneumatic amplifier to higher and lower pressure source, respectively, $l_{4}$ is the opening of outflow valve, $s_{1}, s_{31}, s_{32}$, and $s_{4}$ are the corresponding effective flow area of valve 1 , valve 31 , valve 32 , and outflow valve, and $P_{a}$ is the air pressure around the aircraft. Schedule pressure generator is composed of two components:

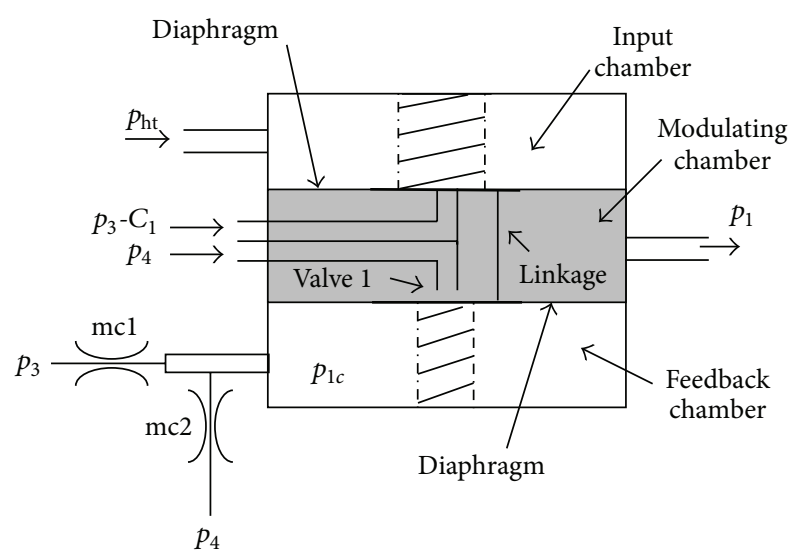

FIGURE 2: Functional structure of differential pressure generator.

proportional pressure generator and differential pressure generator. The two components take part in schedule pressure modulating at different altitude of flight. Proportional pressure generator works at lower altitude of flight, and differential pressure generator takes over its job a height about 5100 meter. The two components have similar structure and similar governing equations. Therefore in the present paper, only differential pressure generator is analyzed. Correspondingly only stability at altitude above 5100 meter is considered. The stability at lower altitude can be analyzed similarly.

The functional structure of differential pressure generator is shown in Figure 2. Having a double nozzle-flapper structure disposed in modulating chamber, differential pressure generator has a similar structure as proportional pressure generator [5]. The differential equation of its valve's opening $l_{1}$ is

$$
\dot{l}_{1}= \begin{cases}\frac{\left(p_{a}-p_{1 c}\right) A_{1}+F_{10}+k_{1} l_{1}}{f_{1}}, & 0<l_{1}<L_{M 1}, \\ 0, & l_{1} \leq 0 \text { or } l_{1} \geq L_{M 1},\end{cases}
$$

where $A_{1}$ is the effective area of pressure applied on diaphragms, $F_{10}$ is the preload spring force on diaphragms, $k_{1}$ is the equivalent elasticity coefficient of the springs, and $f_{1}$ is the kinetic friction coefficient of valve 1 movement.

In the situation of dynamic balance, volumes flowing in and out of feedback chamber are equal. According to flow rate 
calculation formula equation (3) in [6] and equation (1) in [7], $p_{1 c}$ is dynamic solution of the following equation:

$$
q_{c 1} p_{1 c} \sqrt{1-\left(\frac{p_{3} / p_{1 c}-b_{1}}{1-b_{1}}\right)^{2}}=q_{c 2} p_{4} \sqrt{1-\left(\frac{p_{1 c} / p_{4}-b_{2}}{1-b_{2}}\right)^{2}},
$$

where $q_{c 1}$ is the saturation volume flow of capillary pipe mcl, $q_{c 2}$ is the saturation volume flow of capillary pipe mc2, $b_{1}$ is the critical pressure ratio of capillary $\mathrm{mcl}$, and $b_{2}$ is the critical pressure ratio of capillary mc2. Therefore $p_{1 c}$ is a variable which lies between $p_{3}$ and $p_{4}$.

In accordance with (15) in [8], the change rate of $p_{1}$ is

$$
\begin{aligned}
\dot{p}_{1}= & \frac{240 s_{11} p_{4} \sqrt{p_{1}\left(p_{4}-p_{1}\right)}}{V_{1}} \\
& -\frac{240\left(S_{10}-s_{11}\right) p_{1} \sqrt{\left(p_{3}-C_{1}\right)\left(p_{1}-p_{3}+C_{1}\right)}}{V_{1}},
\end{aligned}
$$

where $s_{11}=c \cdot l_{1}$ is the effective flow area of valve 1 and $S_{10}$ is the maximum effective flow area of valve $1 . C_{1}$ is a constant, which stands for the pressure drop of $P_{3} . V_{1}$ is the volume of modulating chamber of differential pressure generator.

2.2.2. Mathematical Model for Rate Limiter. The rate limiter is composed of a capillary pipe mc3 connecting to a gas container. By limits volume flowing through capillary pipe, the change rate of output pressure in gas container is limited. Dynamic equation of $p_{2}$ is as follows:

$$
\dot{p}_{2}= \begin{cases}\frac{q_{c 3} p_{1}}{V_{2}} \sqrt{1-\left(\frac{p_{2} / p_{1}-b_{3}}{1-b_{3}}\right)^{2}}, & p_{2}<p_{1}, \\ -\frac{q_{c 3} p_{2}}{V_{2}} \sqrt{1-\left(\frac{p_{2} / p_{1}-b_{3}}{1-b_{3}}\right)^{2}}, & p_{2}>p_{1},\end{cases}
$$

where $q_{c 3}$ is the saturation volume flow of capillary pipe mc3, $b_{3}$ is the critical pressure ratio of capillary pipe mc3, and $V_{2}$ is the volume of gas container.

2.2.3. Mathematical Model for Pneumatic Amplifier. The functional structure of pneumatic amplifier is shown in Figure 3. It also has a similar structure as proportional pressure generator. In pneumatic amplifier, there are two independent valves: valve 31 and valve 32 . Suppose the

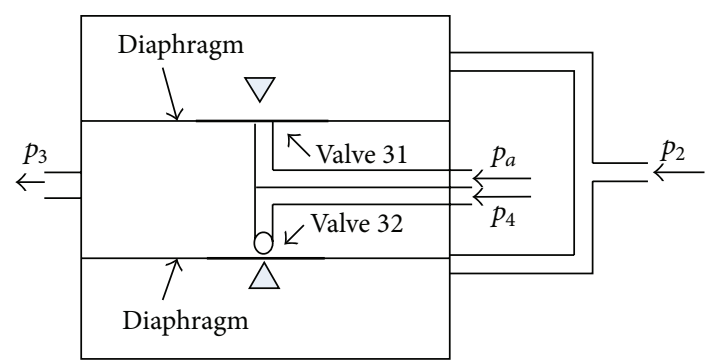

FIGURE 3: Functional structure of pneumatic amplifier.

opening of valve 31 and valve 32 are $l_{31}$ and $l_{32}$, respectively, the relevant differential equations are as follows:

$$
\begin{aligned}
& \dot{l}_{31}= \begin{cases}\frac{\left(p_{2}-p_{3}\right) A_{3}-F_{310}-k_{31} l_{31}}{f_{31}}, & 0<l_{31}<L_{M 31}, \\
0, & l_{31} \leq 0 \text { or } l_{31} \geq L_{M 31},\end{cases} \\
& \dot{l}_{32}= \begin{cases}\frac{\left(p_{3}-p_{2}\right) A_{3}-F_{320}-k_{32} l_{32}}{f_{32}}, & 0<l_{32}<L_{M 32}, \\
0, & l_{32} \leq 0 \text { or } l_{32} \geq L_{M 32},\end{cases} \\
& \dot{p}_{3}= \begin{cases}\frac{240 s_{31} p_{4} \sqrt{p_{3}\left(p_{4}-p_{3}\right)}}{V_{3}}-\frac{120 s_{32} p_{3}}{240 s_{31} p_{4} \sqrt{p_{3}\left(p_{4}-p_{3}\right)}}, & \frac{p_{a}}{V_{3}} \leq 0.5, \\
\frac{V_{3}}{240 s_{32} p_{3} \sqrt{p_{a}\left(p_{3}-p_{a}\right)}} & \frac{p_{a}>0.5,}{V_{3}},\end{cases}
\end{aligned}
$$

where $s_{31}=c \cdot l_{31}$ is the dynamic charging area of valve 31, $s_{32}=c \cdot l_{32}$ is the dynamic discharging area of valve 32 , and $V_{3}$ is the total volume of modulating chamber and pneumatic load of pneumatic amplifier.

\subsubsection{Mathematical Model for Outflow Valve and Cabin} Pressure. Cabin pressure and its change rate is the control object of cabin pressure regulating system. The velocity of the outflow valve opening is

$$
i_{4}= \begin{cases}\frac{\left(p_{4}-p_{3}\right) A_{4}-F_{40}-k_{4} l_{4}}{f_{4}}, & 0<l_{4}<L_{M 4}, \\ 0, & l_{4} \leq 0 \text { or } l_{4} \geq L_{M 4}\end{cases}
$$

where $A_{4}$ is the effective area of air pressure applied on diaphragm of outflow valve, $F_{40}$ is the force preload on outflow valve by reset spring in control chamber of outflow valve, $k_{4}$ is the elasticity coefficient of the reset spring, and $f_{4}$ is the kinetic friction coefficient of outflow valve movement. 
The volume flow rate of outflow valve $G_{\text {Out }}$ is

$$
G_{\text {Out }}= \begin{cases}120 s_{4} p_{4}, & \frac{p_{a}}{p_{4}} \leq 0.5 \\ 240 s_{4} p_{4} \sqrt{\frac{p_{a}}{p_{4}}\left(1-\frac{p_{a}}{p_{4}}\right)}, & \frac{p_{a}}{p_{4}}>0.5 .\end{cases}
$$

According to (13) in [9] and (2) in [10], the change rate of cabin pressure is

$$
\dot{p}_{4}=\frac{G_{S}(h, \dot{h})-G_{L}-G_{\text {Out }}}{V_{C}} \cdot p_{4},
$$

where $V_{C}$ is the volume of aircraft cabin, $G_{L}$ is the volume flow rate caused by cabin leakage, and $G_{S}(h, \dot{h})$ is the volume flow into the cabin caused by air supply system.

\section{Scheme of Stability Analysis and Its Realization}

3.1. Expression of the System in General Form. Set $y_{1}=p_{1}$, $y_{2}=p_{2}, y_{3}=p_{3}, y_{4}=p_{4}, y_{5}=l_{1}, y_{6}=l_{31}, y_{7}=l_{32}$, and $y_{8}=l_{4}$, and according to (1) (8), write their differential expressions in a general form [11], as shown in

$$
\begin{gathered}
\frac{d y_{1}}{d t}=f_{1}\left(t, y_{1}, y_{2}, \ldots, y_{8}\right) \\
\frac{d y_{2}}{d t}=f_{2}\left(t, y_{1}, y_{2}, \ldots, y_{8}\right) \\
\vdots \\
\frac{d y_{8}}{d t}=f_{8}\left(t, y_{1}, y_{2}, \ldots, y_{8}\right),
\end{gathered}
$$

and if the initial condition is given in

$$
y_{1}(0)=\alpha_{1}, \quad y_{2}(0)=\alpha_{2}, \ldots, \quad y_{8}(0)=\alpha_{8},
$$

then stability analysis of the system described by differential equation set (9) with initial condition (10) is the object of the present paper.

3.2. Equilibrium Point of the System. An equilibrium point of system (9) with initial condition (10) is a solution, $\mathbf{y}^{*}=$ $\left(y_{1}{ }^{*}, y_{2}{ }^{*}, \ldots, y_{8}{ }^{*}\right)$, of the following algebraic equation:

$$
\begin{gathered}
f_{1}\left(t, y_{1}{ }^{*}, y_{2}{ }^{*}, \ldots, y_{8}{ }^{*}\right)=0 \\
f_{2}\left(t, y_{1}{ }^{*}, y_{2}{ }^{*}, \ldots, y_{8}{ }^{*}\right)=0 \\
\vdots \\
f_{8}\left(t, y_{1}{ }^{*}, y_{2}{ }^{*}, \ldots, y_{8}{ }^{*}\right)=0 .
\end{gathered}
$$

At certain height (ht), the considered cabin pressure regulating system has only one equilibrium point. Suppose ht is higher than switching height, at which differential pressure component takes over the modulating of schedule pressure. The equilibrium point of the system (9) at height ht is

$$
\begin{gathered}
l_{1}{ }^{*}=\frac{1}{2} L_{\mathrm{Max}} \quad l_{2}{ }^{*}=l_{3}{ }^{*}=0, \\
p_{1}{ }^{*}=p_{2}{ }^{*}=p_{3}{ }^{*}=p_{\mathrm{ht}}+\Delta_{s d}=p_{a}+\Delta_{s d}, \\
p_{4}{ }^{*}=\frac{20\left(F_{10}+p_{\mathrm{ht}}+0.5 k_{1} L_{M 1}\right)}{s}-19 p_{3}{ }^{*} \\
=\frac{20\left(F_{10}+p_{a}+0.5 k_{1} L_{M 1}\right)}{s}-19 p_{3}{ }^{*}, \\
l_{4}{ }^{*}=\frac{\left(p_{4}{ }^{*}-p_{3}{ }^{*}\right) A_{4}-F_{40}}{k_{4}},
\end{gathered}
$$

where $p_{\mathrm{ht}}$ is pressure of atmosphere at height ht, $p_{\mathrm{ht}}=p_{a}$.

3.3. Definition of Stability. The stability in present paper is a kind of global uniform asymptotic stability, and its definition [12] is as follows.

System is said to be asymptotically stable about its equilibrium if it is stable in the sense of Lyapunov, and furthermore, there exists a constant, $\delta=\delta\left(t_{0}\right)>0$, such that

$$
\left\|\mathbf{x}\left(t_{0}\right)\right\|<\delta \Longrightarrow\|\mathbf{x}(t)\| \longrightarrow 0, \quad \text { as } t \longrightarrow \infty .
$$

The asymptotic stability is said to be uniform if the existing constant is independent of $t_{0}$ over $[0, \infty]$ and is said to be global if the convergence $\|\mathbf{x}(t)\| \rightarrow 0$ is independent of the initial state $x$ over the entire domain on which the system is defined.

On the basis of the above definition, the cabin pressure regulating system has its special requirement on stability; that is, the transient must complete within 25 seconds.

3.4. Scheme of Stability Analysis and Its Realization. As stated above, the considered cabin pressure control system is a nonlinear system consisting of 8 differential equations and form of each equation is very complicated. Therefore stability analysis of the system is difficult.

Describing function method, phase plane method, and Lyapunov method are the three methods commonly used to analyze stability of systems. But to a complex system containing multiple nonlinearities, describing function method is very complicated and unique for special problem [13]. Therefore it is not a universal method. Lyapunov method is an important technique that is used for not only analyzing but also designing stability of the system. And it can be applied conveniently to systems containing new control techniques and systems with special characteristics $[14,15]$. But the difficulty of using Lyapunov function lies in founding and screening Lyapunov function. In this circumstance, Lyapunov function methods cannot be used as it is impossible to find a $V$ function. And classic meaning of phase plane method, designed for second order systems, cannot solve the problem too. Therefore in the present paper, a numerical method is proposed and applied constructing phase plane 
trajectories by approximating time-domain solution of the system. Then on this basis, system stability can be analyzed in accordance with phase plane method.

For System (9) with initial condition (10), within certain time interval $0<t<T$, there are 8 functions $y_{1}(t), y_{2}(t), \ldots, y_{8}(t)$ that satisfy each of the differential equations together with all the initial conditions. But it is difficult to find such functions analytically. Therefore we should first approximate $y_{1}(t), y_{2}(t), \ldots, y_{8}(t)$ numerically at specified time intervals. The existence and uniqueness of the time domain solution are assured by Definition 5.16 and Theorem 5.17 in [11].

To approximate time-domain solution of a complex nonlinear system subject to initial condition, the uniqueness of time-domain solution needs to be ensured firstly. Then a suitable numerical algorithm should be selected and adapted for special needs of considered problem. And selected step size should fulfill requirements of numerical stability of the calculation.

Therefore the secondary object of present paper is to propose a new method to construct phase plane numerically, which extends the scope of phase plane analysis from twodimension to multidimension.

If the system of first-order differential equations (9), subject to the initial conditions (10), has a unique solution $y_{1}(t), y_{2}(t), \ldots, y_{m}(t)$, then numerical methods could be used approximating its time-domain solution within certain period of time.

Taking into consideration computational complexity and accuracy, the improved Euler method is selected, and its computational formulas are as follows:

$$
\begin{gathered}
y_{i(n+1)}=y_{i n}+\frac{h}{2}\left(k_{i 1}+k_{i 2}\right), \\
k_{i 1}=f\left(t_{n}, y_{i n}\right), \\
k_{i 2}=f\left(t_{n}+h, y_{i n}+h k_{i 1}\right),
\end{gathered}
$$

for each $n=0,1, \ldots, N, \quad i=1,2, \ldots, 8$,

where $N$ is number of steps needed to completed computation at time interval $[0, T]$.

In order to construct phase plane trajectories for considered cabin pressure regulating system, approximated time domain solution as well as its derivative needs to be acquired. Therefore the computation formula is adapted, as shown in

$$
\begin{gathered}
y_{i(n+1)}=y_{i n}+\frac{h}{2}\left(k_{i 1}+k_{i 2}\right), \\
k_{i 1}=f\left(t_{n}, y_{i n}\right), \\
k_{i 2}=f\left(t_{n}+h, y_{i n}+h k_{i 1}\right), \\
\dot{y}_{i(n+1)}=f\left(t_{n+1}, y_{i(n+1)}\right),
\end{gathered}
$$

for each $n=0,1, \ldots, N, \quad i=1,2, \ldots, 8$.

In present paper an $\mathrm{m}$-file is programmed on MATLAB platform to acquire data needed, according to equation set (15). Use the notation $M y_{i j}$ and $D y_{i j}$ for each $j=0,1, \ldots, N$ and $i=1,2, \ldots, 8$, to denote an approximation to $y_{i}\left(t_{j}\right)$ and its derivative $y_{i}^{\prime}\left(t_{j}\right)$, respectively. Set

$$
M y_{11}=\alpha_{1}, \quad M y_{21}=\alpha_{2}, \ldots, \quad M y_{81}=\alpha_{8}
$$

The process of computation is described as follows.

(1) Set number of cycle $N$ in accordance with step size $h$ and time limit $T, N=T / h$.

(2) Set initial value as

$$
\begin{gathered}
M y_{1,1}=\alpha_{1}, \quad M y_{2,1}=\alpha_{2}, \ldots, \quad M y_{8,1}=\alpha_{8}, \\
D y_{1,1}=f\left(0, M y_{1,1}\right), \quad D y_{2,1}=f\left(0, M y_{2,1}\right), \ldots, \\
D y_{8,1}=f\left(0, M y_{8,1}\right) .
\end{gathered}
$$

(3) While $s$ increase from 1 to $N$, carry out computation from Step 4 to Step 7:

(4)

$$
\left[\begin{array}{c}
k_{11} \\
k_{21} \\
\vdots \\
k_{81}
\end{array}\right]=\left[\begin{array}{c}
D y_{1 s} \\
D y_{2 s} \\
\vdots \\
D y_{8 s}
\end{array}\right]
$$

(5)

$$
\left[\begin{array}{c}
k_{12} \\
k_{22} \\
\vdots \\
k_{82}
\end{array}\right]=\left[\begin{array}{c}
f_{1}\left(t_{s}+h, M y_{s}+h k_{11}\right) \\
f_{2}\left(t_{s}+h, M y_{s}+h k_{21}\right) \\
\vdots \\
f_{8}\left(t_{s}+h, M y_{s}+h k_{81}\right)
\end{array}\right],
$$

$$
\left[\begin{array}{c}
M y_{1(s+1)} \\
M y_{2(s+1)} \\
\vdots \\
M y_{8(s+1)}
\end{array}\right]=\left[\begin{array}{c}
M y_{1 s} \\
M y_{2 s} \\
\vdots \\
M y_{8 s}
\end{array}\right]+\frac{h}{2}\left[\begin{array}{c}
k_{11}+k_{12} \\
k_{21}+k_{22} \\
\vdots \\
k_{81}+k_{82}
\end{array}\right]
$$

$$
\left[\begin{array}{c}
D y_{1(s+1)} \\
D y_{2(s+1)} \\
\vdots \\
D y_{8(s+1)}
\end{array}\right]=\left[\begin{array}{c}
f_{1}\left(t_{s+1}, M y_{1(s+1)}\right) \\
f_{2}\left(t_{s+1}, M y_{2(s+1)}\right) \\
\vdots \\
f_{8}\left(t_{s+1}, M y_{8(s+1)}\right)
\end{array}\right] .
$$

(8) Plot interested ith pair of variable $\left(M y_{i}, D y_{i}\right)$ of acquired data (here $i$ may be any number between 1 and 8). Phase plane trajectories are now constructed, and stability of system with initial-value may be analyzed in accordance with phase plane methods. 
3.5. Convergence of Calculation. Define model equation of absolute stability as linear differential equation:

$$
\begin{aligned}
{\left[\begin{array}{c}
\dot{y}_{1} \\
\dot{y}_{2} \\
\vdots \\
\dot{y}_{8}
\end{array}\right] } & =\left|\begin{array}{cccc}
\frac{\partial f_{1}}{\partial y_{1}} & 0 & \cdots & 0 \\
0 & \frac{\partial f_{2}}{\partial y_{2}} & \cdots & 0 \\
\vdots & \vdots & \ddots & \vdots \\
0 & 0 & \cdots & \frac{\partial f_{8}}{\partial y_{8}}
\end{array}\right|\left[\begin{array}{c}
y_{1} \\
y_{2} \\
\vdots \\
y_{8}
\end{array}\right] \\
& =\left|\begin{array}{cccc}
\lambda_{1} & 0 & \cdots & 0 \\
0 & \lambda_{2} & \cdots & 0 \\
\vdots & \vdots & \ddots & \vdots \\
0 & 0 & \cdots & \lambda_{8}
\end{array}\right|\left[\begin{array}{c}
y_{1} \\
y_{2} \\
\vdots \\
y_{8}
\end{array}\right] .
\end{aligned}
$$

The region of absolute stability for the improved Euler method is $\left|1+h \lambda+(h \lambda)^{2} / 2\right|<1$, and the interval of absolute stability is $-2<h \lambda<0$, for each of $\lambda_{1}, \lambda_{2}, \ldots, \lambda_{8}$.

Set $\lambda_{i X}=\max _{0 \leq t \leq T}\left|\partial f_{i} / \partial y_{i}\right|, \lambda_{X}=\max \left(\lambda_{i X}\right),(i=$ $1,2, \ldots, 8)$; then to ensure the results of calculation approximate to the time-domain solution $y(t)$, the interval of $h$ value is limited by $0<h<2 /\left|\lambda_{X}\right|$.

\section{Results}

The method stated above is applied to construct phase plane trajectories of cabin pressure regulating system with 2 initial conditions.

At height ht $=7000 \mathrm{~m}$, the first initial condition is given as

$$
\begin{array}{rlll}
p_{10}=80, & p_{20}=80, & p_{30}=80, & p_{40}=80.5, \\
l_{10}=0, & l_{310}=0, & l_{320}=0, & l_{40}=0 .
\end{array}
$$

is

And at height $\mathrm{ht}=7000 \mathrm{~m}$, the second initial condition

$$
\begin{aligned}
& p_{10}=78, \quad p_{20}=78, \quad p_{30}=78, \quad p_{40}=79.5 \text {, } \\
& l_{10}=0, \quad l_{310}=0, \quad l_{320}=0, \quad l_{40}=0 .
\end{aligned}
$$

According to Section 3.3, the calculated $\left|\lambda_{X}\right|<308.4$; then the interval of $h$ value is $0<h<0.0064$. Then step size $h$ is chosen as $h=0.001 \mathrm{~s}$. Take into consideration the actual requirement of the system, set $T=30 \mathrm{~s}$, and then $N=$ $T / h=30000$. And data is acquired by an $\mathrm{m}$-file programmed in accordance with computation process listed in Section 3.2.

In the cabin pressure regulating system, pressure stability of $p_{2}$ and $p_{4}$ is variables interested. The phase plane trajectories of $p_{2}$ and $p_{4}$ are shown in Figures 4 and 5, respectively.

Figure 4 shows that, under the 2 given initial conditions, a limit cycle exists in pressure $p_{2}$. That means pressure $p_{2}$ will not be stable. The fluctuation in $p_{2}$ will pass on and

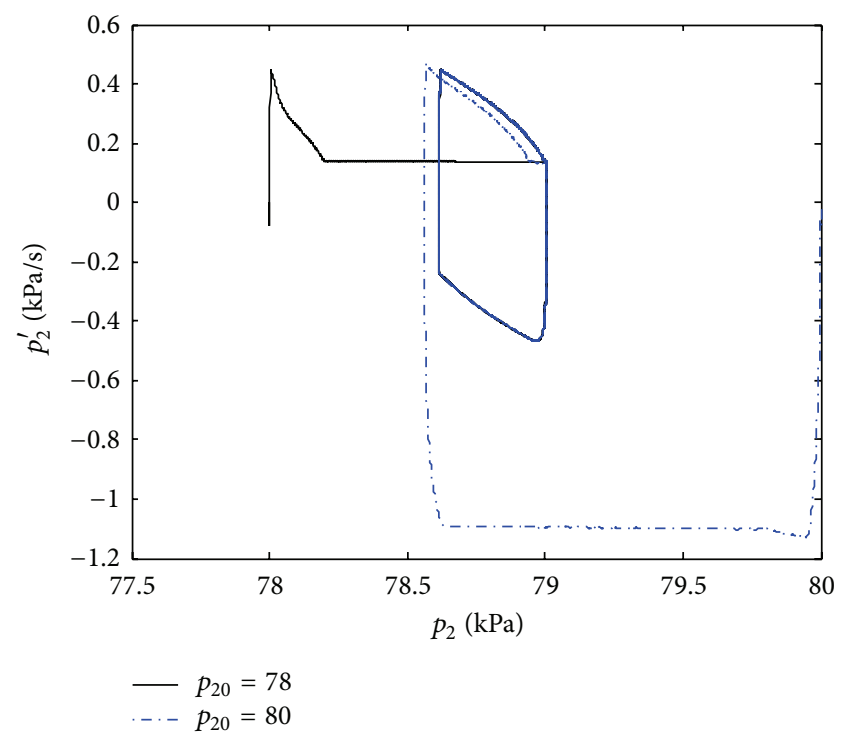

FIgURE 4: Phase plane trajectories of $p_{2}$.

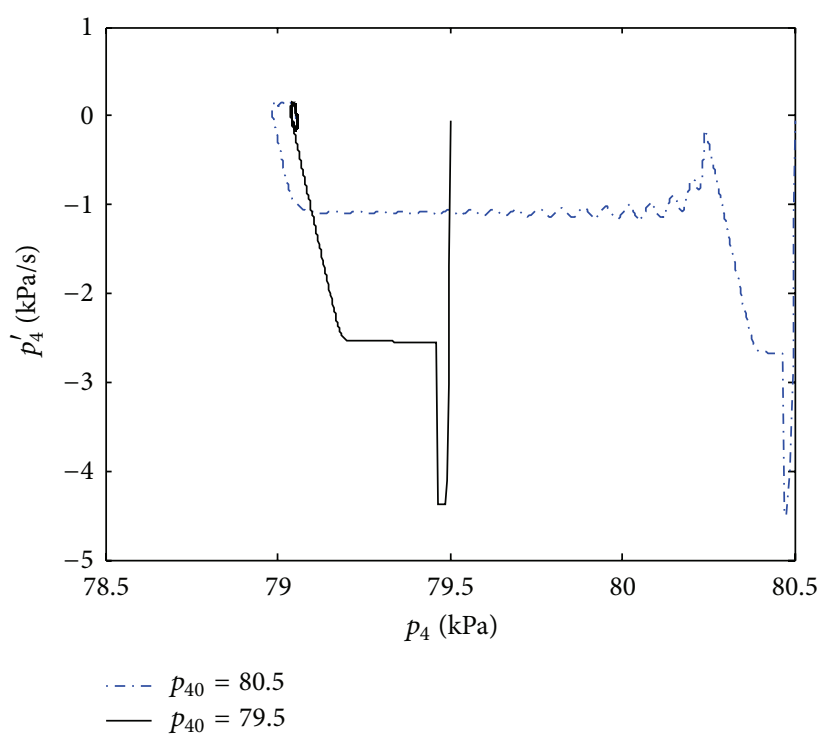

FIgURE 5: Phase plane trajectories of $p_{4}$.

be reproduced in cabin pressure, may be in reduced or comparable amplitude. Figure 5 shows an amplitude reduced limit cycle exists in $p_{4}$ phase plane trajectories. This is not allowed for the fluctuation may be aggravated in the complex environment of the flying aircrafts.

In order to ensure stability of cabin pressure $p_{4}$, the parameters of the system should be adjusted. According to design experience, enhancing energy storage capability in the pressure regulating process helps to realize stability of system. And reducing dead zone threshold of pneumatic amplifier helps in eliminating limit cycle. Therefore, by substituting new type of polymer material for old one, and using new type of spring with bigger elastic coefficient in differential pressure generator, some system parameters are changed. And preload 


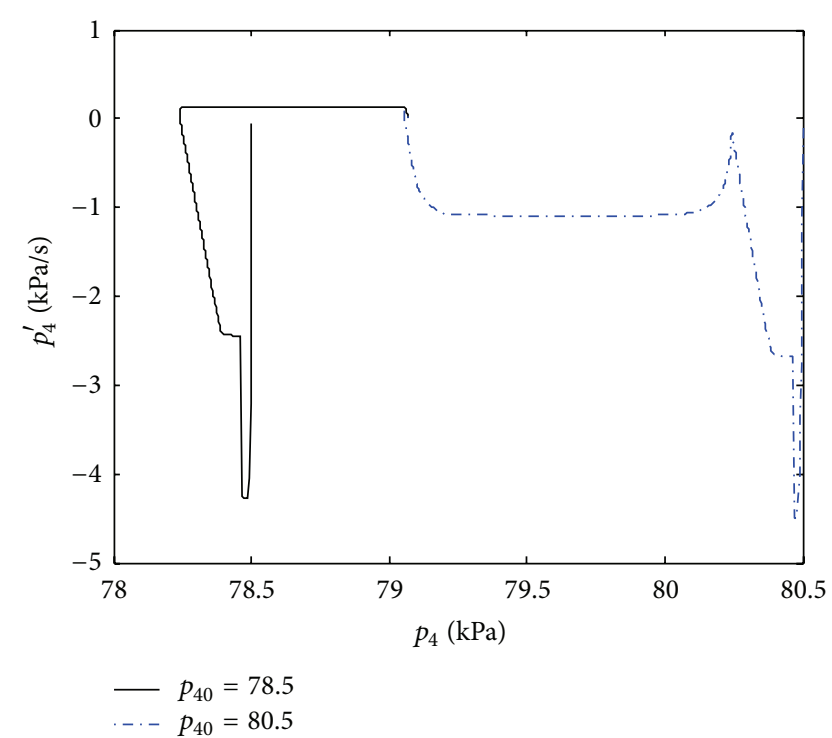

FIGURE 6: Phase plane trajectories of cabin pressure $p_{4}$ after parameters modification.

forces on amplifier's valve are reduced slightly. New set of parameters is as follows:

$$
\begin{gathered}
k_{1}=4 \mathrm{~N} / \mathrm{cm}, \quad k_{31}=0.2 \mathrm{~N} / \mathrm{cm}, \quad k_{32}=0.2 \mathrm{~N} / \mathrm{cm}, \\
f_{1}=0.1, \quad f_{31}=f_{32}=0.1, \quad f_{4}=0.5, \\
F_{1}=9.4 \mathrm{~N}, \quad F_{310}=0.15 \mathrm{~N}, \\
F_{320}=0.15 \mathrm{~N}, \quad F_{4}=1.5 \mathrm{~N} .
\end{gathered}
$$

And the corresponding replaced old set of parameters is as follows:

$$
\begin{gathered}
k_{1}=3.5 \mathrm{~N} / \mathrm{cm}, \quad k_{31}=0.17 \mathrm{~N} / \mathrm{cm}, \\
k_{32}=0.17 \mathrm{~N} / \mathrm{cm}, \\
f_{1}=0.06, \quad f_{31}=f_{32}=0.05, \quad f_{4}=0.5, \\
F_{1}=9.4 \mathrm{~N}, \quad F_{310}=0.2 \mathrm{~N}, \\
F_{320}=0.2 \mathrm{~N}, \quad F_{4}=1.5 \mathrm{~N} .
\end{gathered}
$$

After parameters adjustment, the new phase plane trajectories of $p_{4}$ are shown in Figure 6. Figure 6 shows that no limit cycle exists in the system and the phase plane trajectories goes to the same equilibrium point even though the initial conditions are different. The simulating result shown in Figure 7 confirms this conclusion. Figure 7 shows that the stability of cabin pressure is achieved quickly and little fluctuation will happen. On this base, we come to the conclusion that the system is stable after parameter modification.

It needs to point out that, comparing with twodimensional system, in a multidimensional system some difference exists in their phase plane trajectories; that is, the trajectories may come across limit cycle before it finally enters into it, as shown by Figure 4 .

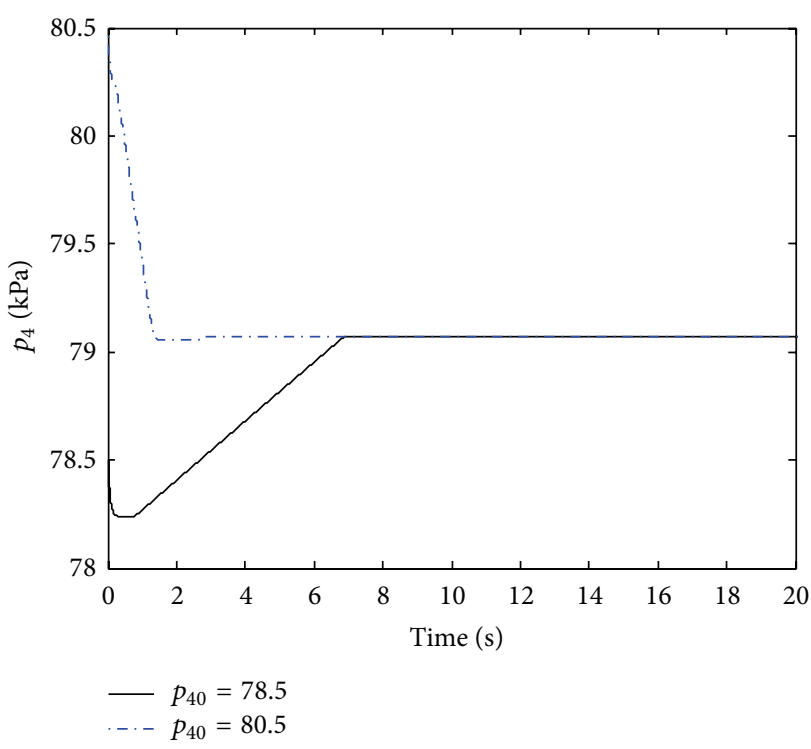

FIGURE 7: Simulation result of cabin pressure $p_{4}$.

\section{Conclusion}

The considered cabin pressure regulating system in present paper is a multidimensional complex nonlinear system. To analyze stability of cabin pressure regulating system which is used in advanced military airplane, a numerical method of construct phase plane trajectories is proposed in the present paper. This method extends the scope of phase plane analysis from two-dimension to multidimension. The method is applied to stability analysis of cabin pressure regulating system, and the validity of analysis is verified by simulation result. The method is applicable to complex nonlinear system of any dimension and may provide an effective way for stability analysis of complex nonlinear systems. It is instructive for researchers facing similar problems.

\section{Nomenclature}

$A_{1}$ : Effective area of pressure applied on diaphragms in differential pressure generator

$A_{3}$ : Effective area of pressure applied on diaphragms of pneumatic amplifier

$A_{4}$ : Effective area of pressure applied on diaphragm of outflow valve

$f_{1}$ : Kinetic friction coefficient of valve 1 movement

$f_{31}$ : Kinetic friction coefficient of valve 31 movement

$f_{32}$ : Kinetic friction coefficient of valve 32 movement

$f_{4}:$ Kinetic friction coefficient of outflow valve

$F_{10}$ : Preload force on diaphragm of differential pressure generator

$F_{310}$ : Preload force on valve 31

$F_{320}$ : Preload force on valve 32

$F_{40}$ : Preload force on outflow valve 
$G_{S}$ : $\quad$ Volume flow rate caused by air supply system

$G_{L}$ : Volume flow rate caused by cabin Leakage

$G_{\text {Out }}$ : Volume flow rate caused by outflow valve

ht: Height of the aircraft

$k_{1}$ : Equivalent elasticity coefficient of springs in differential pressure generator

$k_{31}$ : Equivalent elasticity coefficient of valve 31 movement

$k_{32}$ : Equivalent elasticity coefficient of valve 32 movement

$k_{4}$ : Equivalent elasticity coefficient of the reset spring in outflow valve

$l_{1}$ : Opening of valve 1 in differential pressure generator

$l_{31}$ : Opening of valve 31

$l_{32}$ : Opening of valve 32

$l_{4}$ : Opening of outflow valve

$L_{M 1}$ : Maximum opening of valve 1

$p_{1}$ : Output pressure of differential pressure generator

$p_{2}$ : Output pressure of rate limiter

$p_{3}$ : Output pressure of pneumatic amplifier

$p_{4}$ : Pressure in cabin of airplane

$p_{1 c}$ : Pressure in control chamber of differential pressure generator

$p_{a}:$ Ambient pressure around airplane

$q_{c 1}$ : Saturation volume flow of capillary $\mathrm{mcl}$

$q_{c 2}$ : Saturation volume flow of capillary mc2

$q_{c 3}$ : Saturation volume flow of capillary mc3

$s_{11}$ : Dynamic flow area of valve 1

$s_{31}$ : Dynamic flow area of valve 31

$s_{32}$ : Dynamic flow area of valve 32

$s_{4}$ : Dynamic flow area of outflow valve

$S_{10}$ : Maximum effective flow area of valve 1

$b_{1}$ : Critical pressure ratio of capillary $\mathrm{mcl}$

$b_{2}$ : Critical pressure ratio of capillary mc2

$b_{3}$ : Critical pressure ratio of capillary mc3

$V_{1}$ : Volume of modulating chamber in differential pressure generator

$V_{1 c}$ : Volume of feedback chamber in differential pressure generator

$V_{2}$ : Volume of gas container in rate limiter

$V_{3}$ : Total volume of modulating chamber and pneumatic load of pneumatic amplifier

$V_{4}$ : Volume of cabin.

\section{Conflict of Interests}

The authors declare that there is no conflict of interests regarding the publication of this paper.

\section{References}

[1] SAE Aerospace, "Aircraft cabin pressurization criteria," Tech. Rep. ARP1270B, 2010.

[2] K. Linnet and R. Crabtree, "What is next in commercial aircraft environmental control system," SAE Technical Paper Series 932057, 1993.
[3] B. Alagha, S. Ahmadbeigy, S. A. J. Moosavi, and S. M. Jalali, "Hypoxia symptoms during altitude training in professional iranian fighter pilots," Air Medical Journal, vol. 31, no. 1, pp. 28 32, 2012.

[4] Z. Lei, F. Yongling, and Z. Jingquan, "Research on the controller of the digital cabin pressure regulating system based on FIMF," in Proceedings of the IEEE International Conference on Advanced Computer Control (ICACC '10), vol. 5, pp. 454-458, March 2010.

[5] Z. Xinhua, X. Lili, L. Lizhuo, and Y. Liangpu, "Static characterstics of proponional pressure regulating components for aircrat cabin pressure regulator," Machine Design and Research, vol. 2, pp. 94-96, 104, 2013.

[6] K. Kawashima, Y. Ishii, T. Funaki, and T. Kagawa, "Determination of flow rate characteristics of pneumatic solenoid valves using an isothermal chamber," Transactions of the ASME Journal of Fluids Engineering, vol. 126, no. 2, pp. 273-279, 2004.

[7] K. Oneyama, T. Takahashi, Y. Terashima, K. Kuroshita, and T. Kagawa, "Study and suggestion on flow-rate characteristics of pneumatic components," in Proceedings of the 7th international Symposium on Fluid Control, Measurement and Visualization, 2003.

[8] C. Maolin, "Modern gasdynamics, theory, techniques, and practice, lecture 1: flow-rate characteristics of pneumatic components," Hydraulics Pneumatics \& Seals, vol. 2007, no. 2, pp. 44-48, 2007.

[9] K. Li, W. Liu, J. Wang, and Y. Huang, "An intelligent control method for a large multi-parameter environmental simulation cabin," Chinese Journal of Aeronautics, vol. 26, no. 6, pp. 13601369, 2013.

[10] F. D. Ramos, C. R. de Andrade, and E. L. Zaparoli, "Computational simulation of an aircraft cabin pressure control system," in Proceedings of the 18th International Congress of Mechanical Engineering, Ouro Preto, Brazil, November 2005.

[11] R. L. Burden, Numerical Analysis, Brooks/Cole, Cengage Learning Australia, 2010.

[12] C. Guanrong, "Stability of nonlinear system," in Encyclopedia of $R F$ and Microwave Engineering, pp. 4881-4896, John Wiley \& Sons, New York, NY, USA, 2004.

[13] R. Gran and M. Rimer, "Stability analysis of systems with multiple nonlinearities," IEEE Transactions on Automatic Control, vol. 10, no. 1, pp. 94-97, 1965.

[14] Y. Chen, W. X. Zheng, and A. Xue, "A new result on stability analysis for stochastic neutral systems," Automatica, vol. 46, no. 12, pp. 2100-2104, 2010.

[15] G. Garcia and S. Keshmiri, "Adaptive and resilient flight control system for a small unmanned aerial system," International Journal of Aerospace Engineering, vol. 2013, Article ID 289357, 25 pages, 2013. 

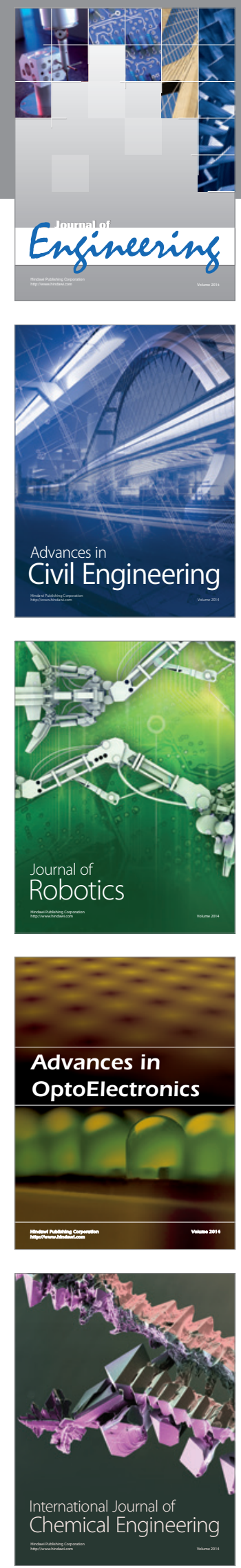

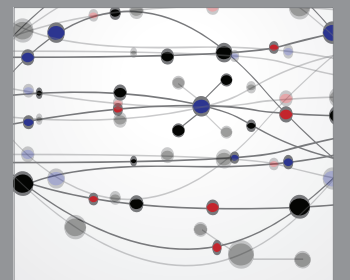

The Scientific World Journal
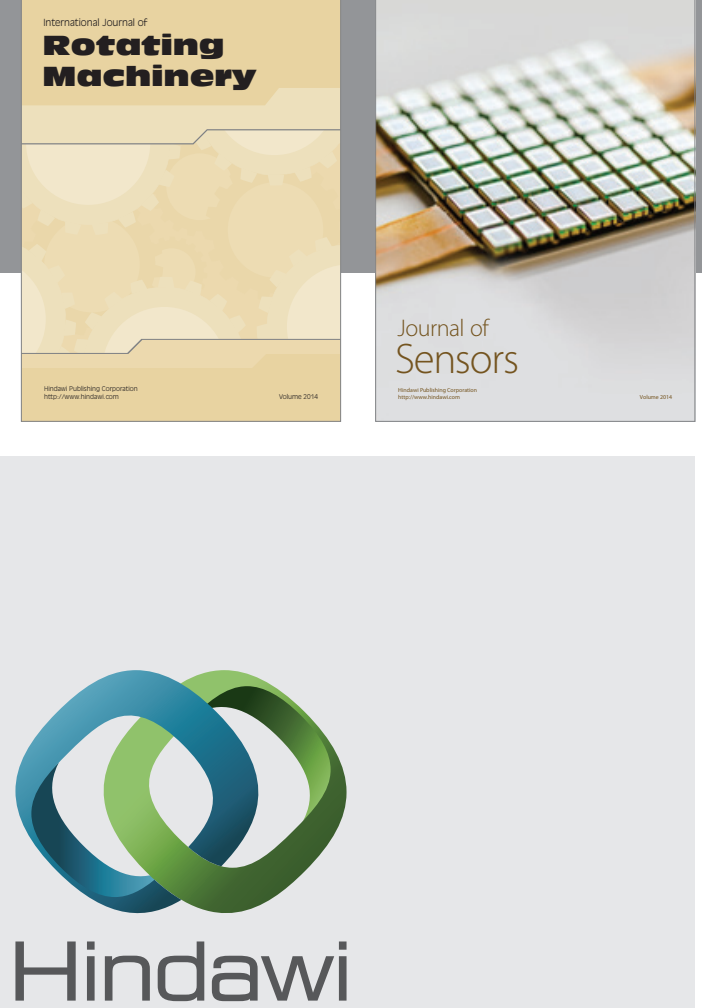

Submit your manuscripts at http://www.hindawi.com
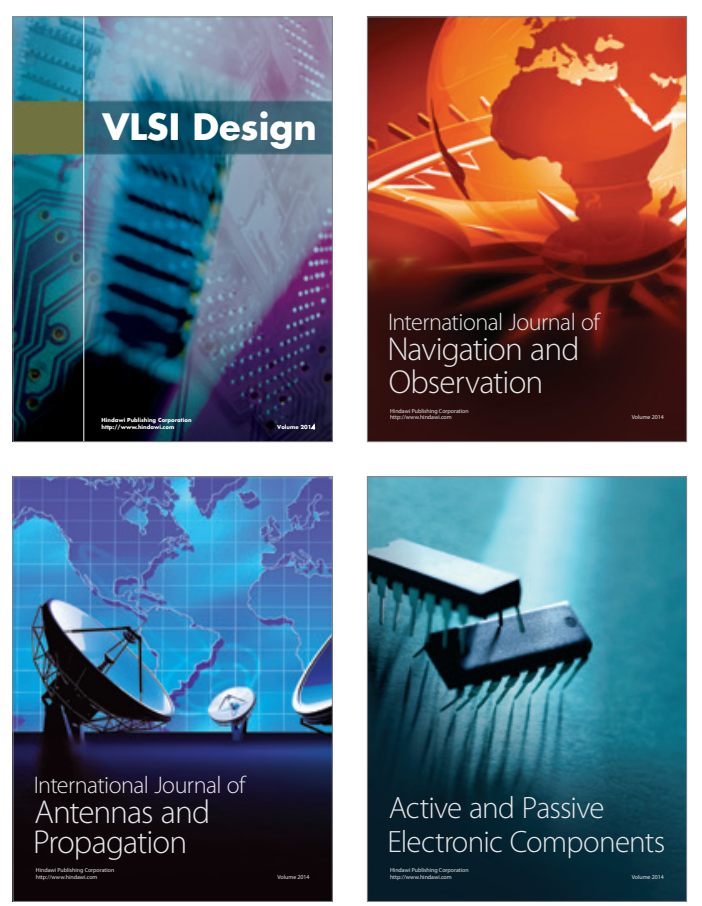
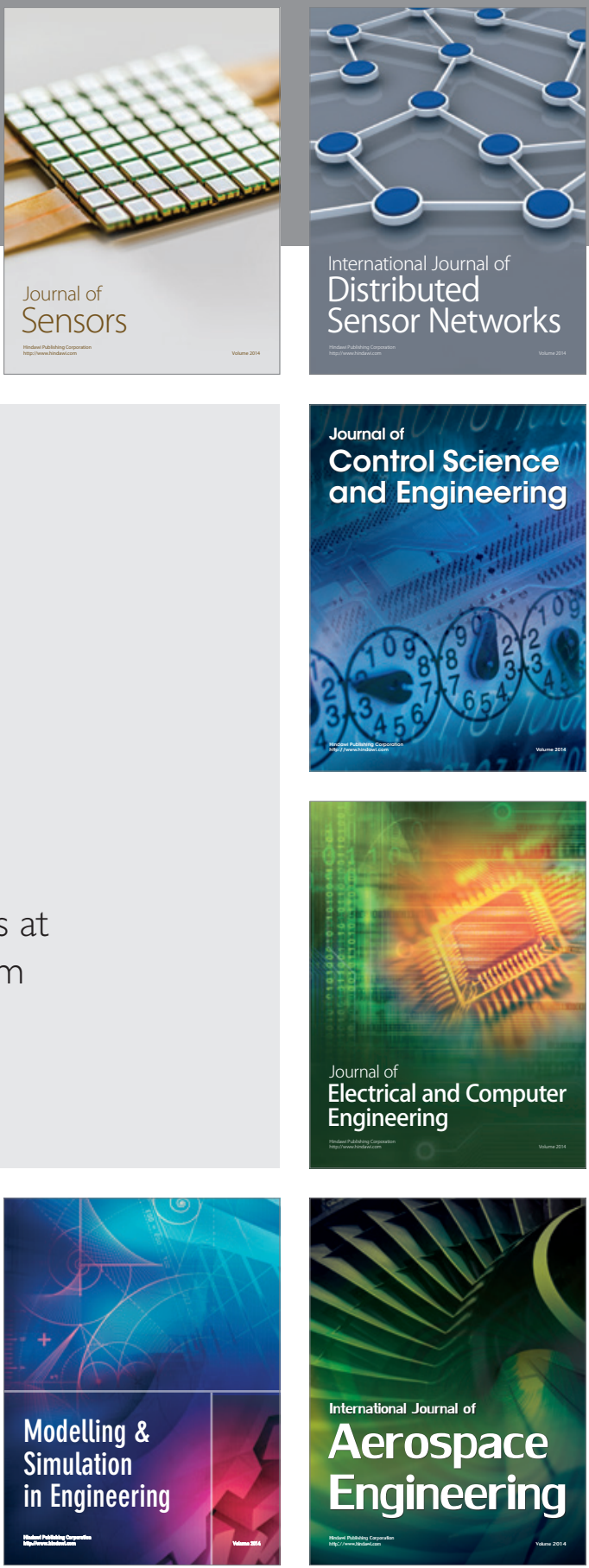

Journal of

Control Science

and Engineering
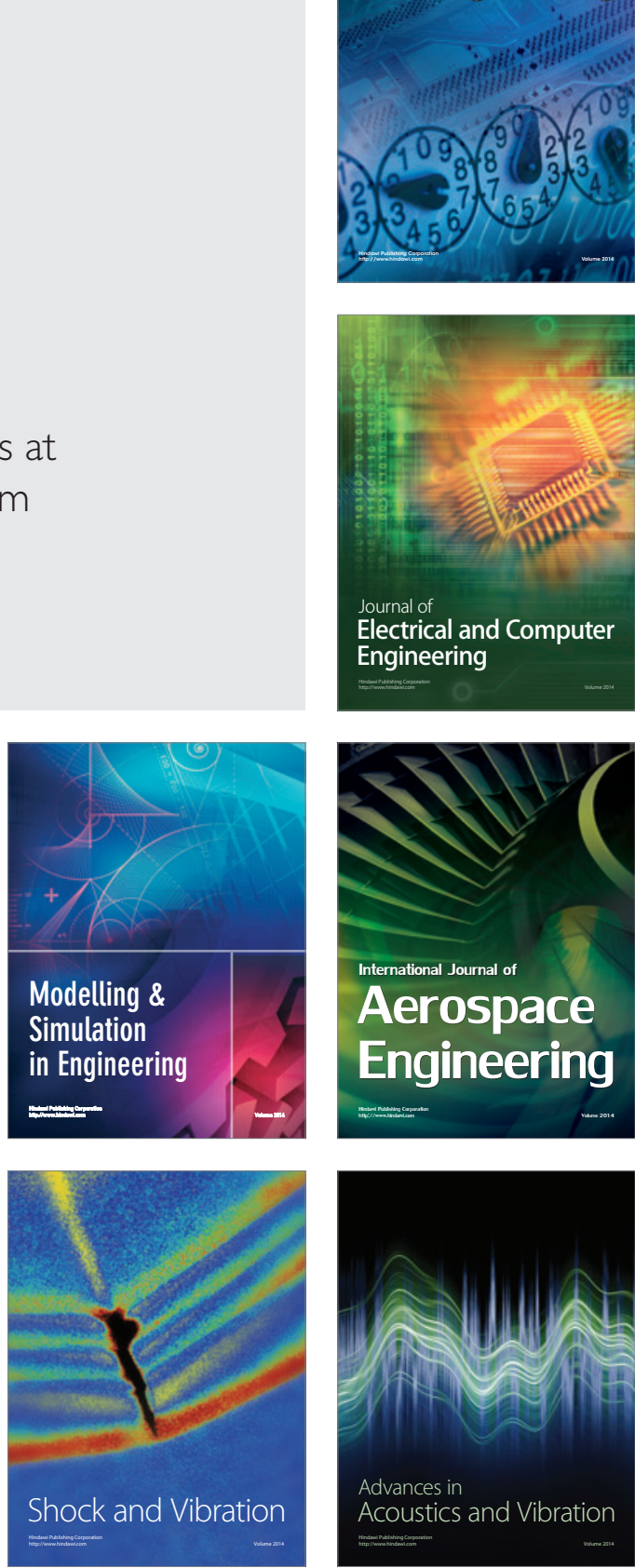